\title{
Fairness Matters: Identification of Active RFID Tags with Statistically Guaranteed Fairness
}

\author{
Muhammad Shahzad* \\ Department of Computer Science \\ North Carolina State University \\ Raleigh, NC, USA \\ mshahza@ncsu.edu
}

\author{
Alex X. Liu \\ Department of Computer Science and Engineering \\ Michigan State University \\ East Lansing, MI, USA \\ alexliu@cse.msu.edu
}

\begin{abstract}
RFID systems with battery powered active tags are widely used in various applications such as supply chain management and object tracking. In RFID identification, tags transmit their IDs to readers over a shared wireless medium; thus, transmissions from tags often collide causing some tags to use their scarce energy resources to retransmit their IDs. Existing RFID identification protocols are unfair in the sense that some tags transmit more times compared to others and thus deplete their batteries faster. Locating tags with depleted batteries for replacement is troublesome. This paper addresses the fundamental problem of ensuring required fairness in the number of transmissions per tag while minimizing identification time in active RFID tag identification. We propose the first Fair RFID Identification Protocol (FRIP) that can achieve any required amount of fairness. The key idea behind FRIP is to bound the expected number of tags that transmit more than once by finding optimal frame sizes for the standardized frame slotted Aloha. We implemented and performed side-byside comparisons of FRIP with all nine major existing RFID identification protocols. Our results show that FRIP can achieve arbitrarily high fairness. FRIP reduces the average number of transmissions per tag by at least 2.62 times compared to the best existing protocol. At the same time, it is faster than the existing protocols. FRIP is easy to deploy because it is compliant with the C1G2 standard, and thus, requires no modifications to tags or to the communication protocol between tags and readers. It only needs to be implemented on readers as a software module. FRIP works with multiple readers.
\end{abstract}

Keywords-RFID; Fairness; Identification;

\section{INTRODUCTION}

\section{A. Motivation and Problem Statement}

Radio Frequency Identification (RFID) system is a wireless system that is comprised of readers and tags. The job of the reader is to read the IDs of the tags and possibly some data appended to the IDs. The job of the tag is to transmit its ID and the appended data to the reader when asked for. A reader has a dedicated power source with significant computing power. It reads the IDs of tags and arbitrates the shared wireless medium among them. There are two types of tags: (1) passive tags, which do not have batteries, are powered up by harvesting the radio frequency

\footnotetext{
* The work of Muhammad Shahzad was conducted while he was a Ph.D. student at Michigan State University under the supervision of Alex X. Liu.
}

energy from readers, and have communication ranges often less than 20 feet; (2) active tags, which have batteries and have communication ranges up to 300 feet.

RFID systems with active tags are widely used in various applications, such as supply chain management [1], object tracking [2], inventory control, electronic toll collection, and access control [3], because active tags have better communication quality and reliability than passive tags. For example, Walmart uses active tags to track expensive clothing merchandize for better inventory control [4] and Honeywell Aerospace uses active tags to track its products from birth through use by airlines and repair by Honeywell [5]. Active tags with embedded sensors have seen increasing adoption in applications such as healthcare [6], supply chain management [1], monitoring server cabinet temperatures [7], and disaster management [8]. An example of active tags with embedded sensors is the Tempcorder series of RFID tags used for underground railways monitoring, industrial hazard prevention, and data center monitoring [9]. In this paper, we deal with active RFID tags.

In the routine task of reading the IDs of active tags, i.e., the process of RFID identification, tags transmit their IDs to readers over a shared wireless medium. Therefore, their transmissions often collide causing some tags to use their scarce energy resources to retransmit their IDs. The transmission of IDs to the readers is the most energy consuming activity of an active tag [10]. RFID tags transmit and receive at a constant power regardless of how close or far they are from the reader. In many scenarios such as railways monitoring, reflection seismology (used by oil exploration companies to determine the subsurface rock structure), and automotive and aircraft assembly lines, the tags have to transmit their IDs every few seconds [11]-[14]. Due to the frequent transmissions, batteries of the tags used in such applications have a lifetime only of the order of a few weeks. If the RFID identification protocol is unfair in the sense that some tags transmit more times than others, then the batteries of those tags deplete faster than others. When the batteries of tags do not deplete at the same time, every few weeks, network operators have to identify and locate tags that have 
depleted batteries so that they can replace their batteries, which is typically a manual and laborious process. In many settings, it can be hard to find tags with depleted batteries because they can not be electronically located anymore. In contrast, if the number of transmissions per tag during identification is roughly equal for all tags, then tag batteries will deplete at roughly the same time and thus the network operators can replace the batteries of all tags at the same time.

This paper addresses the fundamental problem of achieving fairness in RFID identification while minimizing identification time. Specifically, given a tag population of unknown size and a required fairness $\alpha$, design a tag identification protocol that minimizes the identification time under the constraint that the fairness in terms of number of transmissions per tag is no less than $\alpha$. We use the well known Jain's fairness index to quantify fairness [15]. The identification protocol should be compliant with the prevalent EPCGlobal Class 1 Generation 2 (C1G2) RFID standard [16].

\section{B. Limitations of Prior Art}

To the best of our knowledge, no prior work has been targeted on developing a fair RFID identification protocol that can achieve any required fairness. Existing RFID identification protocols mostly focus on minimizing identification time and are unfair because in each round of identification, the same set of tags has to transmit more times compared to the other tags. In [17], Shahzad and Liu evaluated the fairness of their TH protocol along with other RFID identification protocols, and showed that $\mathrm{TH}$ achieves the highest fairness among all existing protocols. However, TH is not designed to achieve any required fairness; its design objective is to just minimize the identification time without fairness constraints. In contrast, our objective is to minimize the identification time with fairness constraints. The maximum value of Jain's fairness Index that TH achieves is only 0.84 . To understand what fairness of 0.84 means, consider a population of active tags, where the battery of each tag lasts for a hundred thousand transmissions, and a reader performs one thousand identification rounds each day. For a protocol with fairness of 0.84 , the batteries of $20 \%$ tags will last for 33 days, of another $30 \%$ tags will last for 50 days, and of the remaining 50\% will last for about 100 days. In comparison, with fairness of 0.99 , the batteries of $99 \%$ tags will last for 100 days, and only $1 \%$ will deplete in 50 days. Even though the batteries of $1 \%$ tags will deplete in 50 days, they wont usually need to be found and replaced because in large scale deployments, e.g., in railways monitoring, loss of up to $10 \%$ tags is tolerable because the remaining tags will still provide data that is enough to ensure smooth operation. This tolerability of $10 \%$ is application specific, but usually in most applications, the loss of up to 5\% tags is tolerable.

\section{Proposed Approach}

In this paper, we propose FRIP, the first Fair $\underline{R} F I D$ Identification Protocol. The communication protocol used by
FRIP is the C1G2 standardized frame slotted Aloha protocol, in which a reader first broadcasts a value $f$ to the tags in its vicinity, where $f$ represents the number of time slots present in a forthcoming frame and lies in the range $1 \leq f \leq 2^{15}$. Each $\mathrm{C} 1 \mathrm{G} 2$ compliant tag has an inventory bit, which is initialized to 0 . Each tag whose inventory bit is 0 randomly picks a time slot in the frame and transmits during that slot. If no tag transmits in a slot, it is called an empty slot; if exactly one tag transmits, it is called a singleton slot; and if two or more tags transmit, it is called a collision slot. In a singleton slot, the reader successfully gets the ID of the transmitting tag and issues a command to the tag to change its inventory bit to 1 . The reader executes several frames until all slots in the frame are empty slots, which indicates that all tags have been identified. We assume that the communication channel between readers and tags is reliable i.e., tags correctly receive queries from the readers and the readers correctly receive responses from the tags. We further assume that each tag in the population is covered by at least one reader during the identification rounds.

The key idea behind FRIP is to bound the expected number of tags that transmit more than once by finding the optimal frame sizes. Such frame sizes ensure that the required fairness is achieved while minimizing the identification time. FRIP optimally trades-off the identification time for fairness.

1) Identification Process: FRIP performs identification in three steps. First, it estimates the size of the RFID tag population. Second, based on the estimate, it calculates the optimal frame size and executes an Aloha frame of this size. Third, after executing the frame, it re-estimates the size of remaining unidentified tag population, uses it to recalculate a new optimal frame size, and executes another frame. FRIP repeats this until it has identified all tags.

Population Size Estimation: To estimate the size of tag population, FRIP uses ART, an estimation scheme proposed by Shahzad and Liu in [18]. We chose ART because of its proven reliability, speed, C1G2 compliance, and ability to estimate sizes of arbitrarily large tag populations. ART uses the frame slotted Aloha protocol and thus, integrates seamlessly with FRIP. To obtain an estimate, ART executes several Aloha frames and obtains a binary sequence of $0 \mathrm{~s}$ and $1 \mathrm{~s}$ from each frame by representing empty slots in the frame with $0 \mathrm{~s}$ and singleton and collision slots with $1 \mathrm{~s}$. It then calculates the average run size of $1 \mathrm{~s}$ in the binary sequences, which is a monotonically increasing function of the size of tag population, and uses it to estimate the size of tag population.

Calculating Optimal Frame Size: To calculate the optimal frame size, we first express Jain's fairness index as a function of the expected number of singleton slots in each frame. We then express the expected number of singleton slots in any given frame as a function of ratio of unidentified tag population size and frame size. Using 
these two expressions, FRIP calculates an upper bound for the ratio of unidentified tag population size and frame size that should not be exceeded in order to achieve the required fairness. It then calculates the optimal value of this ratio that minimizes the expected identification time under the constraint that the ratio does not exceed the upper bound. As the estimate of unidentified tag population size is known at the start of each frame, FRIP uses the optimal value of the ratio to calculate the optimal size for each frame. FRIP calculates the optimal value of the ratio only once at the start of the identification process and uses this value to calculate all frame sizes throughout the process.

Re-estimation: After each frame, FRIP estimates the size of remaining unidentified tag population by subtracting the number of successfully identified tags at the end of the frame from the number of unidentified tags at the start of that frame. Note that FRIP does not execute ART again to reestimate the size of tag population. It executes ART only once at the start of the identification process. It recalculates the optimal size for the next frame by dividing the optimal value of the ratio by the number of remaining unidentified tags and executes the next frame. It continues this process until all slots in a frame turn out as empty slots.

2) Large Frame Size Implementation: For large populations, the value of the optimal frame size may exceed the upper limit of $2^{15}$ slots per frame specified in the C1G2 standard. If this happens, FRIP uses SELECT command, standardized in the C1G2 standard, to specify a subset of tag population that should participate in the identification process in any given frame. Thus, FRIP virtually divides the entire population into smaller groups of roughly equal sizes and then identifies the tags in each group independently. This allows FRIP to use frame sizes $\leq 2^{15}$.

3) Multiple Readers: An application with a large number of RFID tags requires multiple readers with overlapping regions because a single reader may not be able to cover all tags. The use of multiple readers introduces several new types of collisions such as reader-reader collisions and reader-tag collisions. Such collisions can be handled by reader scheduling protocols such as those proposed in [19], [20]. FRIP is compatible with all of these reader scheduling protocols.

\section{Key Novelty and Contributions}

The key novelty of this paper lies in proposing FRIP, an RFID identification protocol that statistically guarantees to achieve any required fairness. The key contributions of this paper are in the mathematical development behind FRIP in making it fair as well as the extensive evaluation and comparison of FRIP with existing protocols. For mathematical development, we formulated the fair identification problem as a constraint optimization problem and solved it optimally. For evaluation and comparison, we implemented FRIP and nine RFID identification protocols, which represent the stateof-the-art, in Matlab. These nine protocols are Aloha [21],
BS [22], ABS [23], TH [17], TW [24], ATW [25], STT [26], MAS [27], and ASAP [28]. We performed side-byside comparisons of FRIP with these protocols using tag populations ranging in sizes from a hundred tags to a million tags and following different distributions. Our results show that FRIP always achieves the required fairness, whereas the fairness value of the best existing protocol, $\mathrm{TH}$, is only 0.84 . FRIP reduces the average number of transmissions per tag by at least 2.62 times compared to the best existing protocol. It also reduces the average energy consumption per tag by at least $11 \%$ compared to the best existing protocol. In terms of identification time, FRIP is faster than all existing protocols except $\mathrm{TH}$ when the values of required fairness in FRIP are set equal to the fairness values achieved by the corresponding existing protocols. Although $\mathrm{TH}$ is faster than FRIP by $13.9 \%$, it can not achieve arbitrarily high fairness.

\section{RELATED WORK}

Ferrero et al. proposed Fair Neighbor Friendly Reader Anticollision protocol (FNFRA) that focuses on increasing fairness in throughput of RFID readers in multiple readers environment [29]. FNFRA increases the fairness in throughput by appropriately adjusting the duration of time each reader gets to identify tags in its coverage region such that the number of tags each reader identifies in its allocated time duration is equal. Note that the objectives of FNFRA and FRIP are fundamentally different: FNFRA focuses on making reader scheduling process fair while FRIP focuses on making the tag identification process fair. To the best of our knowledge, no work has been done towards proposing a protocol to make the tag identification process fair. Existing identification protocols mostly focus on minimizing the identification time and can be divided into three types: (1) deterministic, (2) nondeterministic, and (3) hybrid.

A. Deterministic Identification Protocols

Existing such protocols are based on TW protocol [24]. In TW, a reader first queries 0 and all tags whose IDs start with 0 transmit. If result of the query is a successful read (i.e., exactly one tag transmits) or an empty read (i.e., no tag transmits), the reader queries 1 and all tags whose IDs start with 1 transmit. If the result of the query is a collision, the reader generates two new query strings by appending a 0 and a 1 to the previous query string and queries the tags with these new query strings. All the tags whose IDs start with the new query string transmit. This process continues until all the tags are identified. TW based protocols incur large number of collisions resulting in lack of fairness in each round of identification. Note that if the tag population does not change significantly, the tags that experienced comparatively more collisions in previous round of identification experience more collisions in the next round of identification as well. Therefore, the batteries of the tags that experience more collisions in each round deplete at a faster rate compared to the batteries of the tags that 
experience less collisions. Although several protocols such as STT [26], ATW [25], and TH [17], have been proposed to reduce the number of collisions, they focus on reducing the identification time and do not address the fairness issue. Token-MAC is a deterministic protocol, not based on TW, in which a reader provides tokens, i.e., permission to transmit, to tags such that tags with lower historical success rate receive more tokens [30].

\section{B. Nondeterministic Identification Protocols}

Existing such protocols are either based on frame slotted Aloha [21] or Binary Splitting (BS) [22]. Frame slotted Aloha was described in Section I-C. In BS, the reader asks the tags to transmit their IDs. If more than one tag transmit, BS randomly divides and subdivides the population into smaller groups until each group has only one or no tag. This process of subdivision incurs a lot of collisions resulting in lack of fairness in tag transmissions. ABS is a BS based protocol that is designed for continuous identification of tags [23]. CP is another nondeterministic protocol in which tags cooperate and relay transmissions of neighboring tags to the reader [31]. CP is not based on Aloha or BS.

\section{Hybrid Identification Protocols}

Hybrid protocols combine features from both nondeterministic and deterministic protocols. There are two major such protocols: Multi slotted scheme with Assigned Slots (MAS) [27] and Adaptively Splitting-based Arbitration Protocol (ASAP) [28]. MAS is a TW-based protocol in which each tag that matches the reader's query picks up one of the $f$ time slots to transmit. ASAP divides and subdivides the tag population into subsets until the size of each subset is below a certain threshold and then applies Aloha on each subset. Both these protocols also aim to minimize the identification time and do not address the fairness issue.

\section{Optimal Frame Size}

FRIP calculates optimal frame size at the start of each frame to ensure that the required amount of fairness in number of transmissions per tag is achieved. It uses the estimate of the unidentified tag population size at the start of each frame to calculate the optimal frame size. To obtain expressions for calculating the optimal frame size, we first express Jain's fairness index as a function of the expected number of singleton slots in each frame. Second, we express the time to identify all tags in the population in terms of number of singleton slots, collision slots, and empty slots in each frame. Third, we express the expected number of singleton, collision, and empty slots in any given frame as a function of the ratio of unidentified tag population size and frame size. Fourth, we calculate the expected number of frames that FRIP has to execute to identify all tags in the population. Last, we substitute the expressions of expected values obtained from third and fourth steps into the expressions obtained from first and second steps, and formulate the problem as a constraint optimization problem. The objective of this optimization problem is to minimize the expected identification time under the constraint that the fairness in the number of transmissions per tag is at least equal to the required fairness. More specifically, using the substituted expression from the first step, we calculate an upper bound on the ratio of unidentified tag population size and frame size. Then using the substituted expression from the second step, we calculate the optimal value of this ratio that minimizes the expected identification time under the constraint that the ratio does not exceed the upper bound. As the estimate of unidentified tag population size is known at the start of each frame, FRIP divides it by the optimal value of this ratio to calculate the optimal size for each frame. FRIP is computationally inexpensive because it calculates the optimal value of the ratio only once at the start of the identification process and uses it for all frames throughout the identification process. Next, we explain these five steps followed by a strategy to execute frame sizes that are larger than $2^{15}$. Table I lists the symbols used in this paper.

Table I

SYMBOLS USED IN THE PAPER

\begin{tabular}{|c|l|}
\hline Symbol & \multicolumn{1}{|c|}{ Description } \\
\hline$t$ & estimated \# of tags in the population \\
\hline$t_{i}$ & \# of unidentified tags at start of $i^{\text {th }}$ frame \\
\hline$f_{i}$ & size of $i^{\text {th }}$ frame \\
\hline$f_{\max }$ & max. value of $2^{15}$ for $f_{i}$ allowed by the C1G2 \\
\hline $\mathcal{J}$ & Jain's fairness index \\
\hline$\alpha$ & required fairness \\
\hline$x_{l}$ & \# of times tag with label $l$ transmists \\
\hline$s_{i}, c_{i}, e_{i}$ & singleton, collision, and empty slots in $i^{\text {th }}$ frame \\
\hline$S_{i}, C_{i}, E_{i}$ & $\begin{array}{l}\text { random var. for \# of singleton, collision, and } \\
\text { empty slots in } i^{\text {th }} \text { frame }\end{array}$ \\
\hline$X_{j}$ & indicator random var. for $j^{\text {th }}$ slot to be singleton \\
\hline$Y_{j}$ & indicator random var. for $j^{\text {th }}$ slot to be empty \\
\hline$n$ & expected \# of frames to identify all tags \\
\hline$T_{s}, T_{c}, T_{e}$ & time duration of singleton, coll., and empty slots \\
\hline $\mathcal{T}$ & total identification time \\
\hline$k$ & $t_{i} / f_{i}$ \\
\hline$k_{\min }$ & value of $k$ for which $E[\mathcal{T}]$ is minimum \\
\hline$m$ & upper bound on $k$ \\
\hline$\mu$ & KKT multiplier \\
\hline$E[]$. & expected value operator \\
\hline
\end{tabular}

\section{A. Jain's Fairness Index}

Jain et al. introduced an index, called Jain's fairness index, to rate the fairness in allocation of a resource to a set of users/devices [15]. This index has become the most commonly used metric to measure fairness in communication systems. Let $t$ represent the total number of devices sharing a resource and $x_{l}$ be the portion of the resource allocated to $l^{\text {th }}$ device. The expression for Jain's fairness index is:

$$
\mathcal{J}=\frac{\left(\sum_{l=1}^{t} x_{l}\right)^{2}}{t \times \sum_{l=1}^{t} x_{l}^{2}}
$$

The value of this fairness index ranges from $1 / t$ (worst case when one user gets all the resource and remaining $t-1$ users get nothing) to 1 (best case when all users get equal share of the resource). 
We adapt Jain's fairness index in Equation (1) to measure fairness in the number of transmissions per tag by expressing it as a function of the expected number of singleton slots in each frame. Let $t$ represent the total number of tags in the population. We arbitrarily assign a unique label $l$ to each tag, where $1 \leq l \leq t$. Let $x_{l}$ represent the number of times tag with label $l$ transmits its ID during the identification process. Ideally the value of $x_{l}$ should be same for all values of $l$, which will result in maximum fairness of 1 . Recall that a reader executes several Aloha frames to identify all tags in the population. Let $s_{i}$ represent the number of singleton slots in $i^{\text {th }}$ frame, i.e., $s_{i}$ tags are successfully identified in $i^{\text {th }}$ frame. As per the Aloha protocol, a tag transmits its ID once in each frame until it is successfully identified. This means that a tag that is identified in the $i^{\text {th }}$ frame, transmitted its ID $i$ times. Let $n$ represent the expected number of frames that the reader will execute to identify all the tags in the population. The summation term in the numerator of Equation (1), which gives the sum of number of transmissions by each of the $t$ tags during the identification process, becomes $\sum_{l=1}^{t} x_{l}=s_{1} \times 1+s_{2} \times$ $2+s_{3} \times 3+\ldots+s_{n} \times n$. Similarly, the summation term in the denominator of Equation (1), which gives the sum of square of number of transmissions by each tag, becomes $\sum_{l=1}^{t} x_{l}^{2}=s_{1} \times 1^{2}+s_{2} \times 2^{2}+s_{3} \times 3^{2}+\ldots+s_{n} \times n^{2}$. Thus, Jain's fairness index can be represented as:

$$
\mathcal{J}=\frac{\left(\sum_{i=1}^{n} s_{i} \times i\right)^{2}}{t\left(\sum_{i=1}^{n} s_{i} \times i^{2}\right)}
$$

\section{B. Total Identification Time}

We express the time to identify all tags in a population of size $t$ in terms of number of singleton slots, collision slots, and empty slots in each frame. The total identification time is given by the sum of the time taken by singleton slots, collision slots, and empty slots in all $n$ frames. Let $s_{i}$, $c_{i}$, and $e_{i}$ represent the number of singleton, collision, and empty slots, respectively, in $i^{\text {th }}$ frame. Let $T_{s}, T_{c}$, and $T_{e}$ be the absolute time durations of singleton, collision, and empty slots, respectively. The total identification time $\mathcal{T}$ is given by:

$$
\mathcal{T}=\sum_{i=1}^{n}\left(s_{i} \times T_{s}+c_{i} \times T_{c}+e_{i} \times T_{e}\right)
$$

where $s_{i}+c_{i}+e_{i}=f_{i}$, and $f_{i}$ is the size of $i^{\text {th }}$ frame.

\section{Expected Values of Slots}

We express the expected values of singleton, collision, and empty slots in $i^{\text {th }}$ frame as a function of ratio $t_{i} / f_{i}$, where $t_{i}$ is the size of unidentified tag population at the start of the $i^{\text {th }}$ frame and $f_{i}$ is the size of the $i^{\text {th }}$ frame. Before formally deriving expressions for these expected values, we state an assumption that we use to make the formal development tractable.
We assume that instead of picking a single slot to transmit at the start of $i^{\text {th }}$ frame of size $f_{i}$, a tag independently decides to transmit in each slot of the frame with probability $1 / f_{i}$ regardless of its decision about previous or forthcoming slots. Vogt first used this assumption for the analysis of frame slotted Aloha protocol for RFID and justified its use by recognizing that this problem belongs to a class of problems called occupancy problem, which deals with the allocation of balls to urns [32]. Ever since, the use of this assumption has become a norm in the formal analysis of all Aloha based RFID protocols [18], [21], [32], [33].

The implication of this assumption is that a tag can end up choosing more than one slots in the same frame or even not choosing any at all, which is not in accordance with the $\mathrm{C} 1 \mathrm{G} 2$ standard that requires a tag to pick exactly one slot in a frame. However, this assumption does not create any problems because the expected number of slots that a tag chooses in a frame is still one. The analysis with this assumption is, therefore, asymptotically the same as that without this assumption. Bordenave et al. further explained in detail why this independence assumption in analyzing Aloha based protocols provides results just as accurate as if all the analysis was done without this assumption [34]. Note that this independence assumption is made only to make the formal development tractable. All simulations in the paper are based on the $\mathrm{C} 1 \mathrm{G} 2$ standard where a tag chooses exactly one slot at the start of each frame.

Lemma 1 gives the expressions for expected value of number of singleton, collision, and empty slots.

Lemma 1: Let $S_{i}, C_{i}$, and $E_{i}$ be the random variables representing number of singleton, collision, and empty slots, respectively, in the $i^{\text {th }}$ frame. Let the size of the $i^{\text {th }}$ frame be $f_{i}$. Let $t_{i}$ represent the number of unidentified tags in the population at the start of the $i^{\text {th }}$ frame. Let $k$ be the ratio of $t_{i}$ to $f_{i}$ for all $1 \leq i \leq n$, i.e., $k=t_{i} / f_{i}$. Then

$$
\begin{aligned}
& E\left[S_{i}\right]=k f_{i} e^{-k} \\
& E\left[C_{i}\right]=f_{i}-k f_{i} e^{-k}-f_{i} e^{-k} \\
& E\left[E_{i}\right]=f_{i} e^{-k}
\end{aligned}
$$

Proof: Let $j$ be any arbitrary slot in the frame, where $1 \leq j \leq f_{i}$. Let $X_{j}$ be an indicator random variable for the event when $j^{\text {th }}$ slot is a singleton slot, i.e., $X_{j}=1$ if exactly one tag transmits in the $j^{\text {th }}$ slot, otherwise $X_{j}=0$. The value of $S_{i}$ in terms of $X_{j}$ is therefore given by $S_{i}=\sum_{j=1}^{f_{i}} X_{j}$. The number of tags that transmit in any given slot of a frame follow a binomial distribution. Therefore,

$$
E\left[X_{j}\right]=P\left\{X_{j}=1\right\}=\left(\begin{array}{c}
t_{i} \\
1
\end{array}\right)\left(\frac{1}{f_{i}}\right)\left(1-\frac{1}{f_{i}}\right)^{t_{i}-1} \approx k e^{-k}
$$

As $\left\{X_{1}, X_{2}, \ldots, X_{f_{i}}\right\}$ forms a set of identically distributed random variables, the expected value of $S_{i}$ is given by $E\left[S_{i}\right]=E\left[\sum_{j=1}^{f_{i}} X_{j}\right]=f_{i} \times E\left[X_{j}\right]=k f_{i} e^{-k}$.

Similarly, let $Y_{j}$ be an indicator random variable for the event when $j^{\text {th }}$ slot is an empty slot, which implies that 


$$
\begin{aligned}
& E_{i}=\sum_{j=1}^{f_{i}} Y_{j} \text {. Therefore, } \\
& E\left[Y_{j}\right]=P\left\{Y_{j}=1\right\}=\left(\begin{array}{c}
t_{i} \\
0
\end{array}\right)\left(\frac{1}{f_{i}}\right)^{0}\left(1-\frac{1}{f_{i}}\right)^{t_{i}} \approx e^{-k}
\end{aligned}
$$

Thus, the expected value of $E_{i}$ becomes $E\left[E_{i}\right]=$ $E\left[\sum_{j=1}^{f_{i}} Y_{j}\right]=f_{i} \times E\left[Y_{j}\right]=f_{i} e^{-k}$. As $C_{i}=f_{i}-S_{i}-E_{i}$, $E\left[C_{i}\right]=f_{i}-E\left[S_{i}\right]-E\left[E_{i}\right]=f_{i}-k f_{i} e^{-k}-f_{i} e^{-k}$.

We use the expressions for $E\left[S_{i}\right], E\left[C_{i}\right]$, and $E\left[E_{i}\right]$ as values of $s_{i}, c_{i}$, and $e_{i}$, respectively, i.e., $s_{i}=E\left[S_{i}\right]$, $c_{i}=E\left[C_{i}\right]$, and $e_{i}=E\left[E_{i}\right]$. Figure 1 shows the theoretically calculated expected values of number of singleton, collision, and empty slots from Equations (4), (5), and (6), and experimentally observed values of $s_{i}, c_{i}$, and $e_{i}$ from simulations. The continuous lines in the figure are the theoretical plots and the dots are the experimentally observed values from simulations. We make two conclusions from Figure 1. First, our independence assumption does not cause the theoretical analysis to deviate from practically observed values. Second, we can use $s_{i}=E\left[S_{i}\right], c_{i}=E\left[C_{i}\right]$, and $e_{i}=E\left[E_{i}\right]$.

\section{Expected Number of Aloha Frames}

Next, we calculate the expected number of frames $n$ that FRIP executes to identify all tags in the RFID tag population. Lemma 2, gives the expression for $n$.

Lemma 2: Let $k$ be the ratio of number of unidentified tags in the population at the start of any given frame to the size of that frame. Let size of the population be $t$. The expected number of frames, $n$, that FRIP executes to identify these $t$ tags is given by:

$$
n=-\frac{\ln \{t\}}{\ln \left\{1-e^{-k}\right\}}
$$

Proof: Recall that $t_{i}$ represents the number of unidentified tags at the start of $i^{\text {th }}$ frame. The value of $t_{i}$ can be calculated iteratively using the equation $t_{i}=t_{i-1}-s_{i-1}$, where $t_{1}=t$. As $s_{i-1}=E\left[S_{i-1}\right]=k f_{i-1} e^{-k}=\frac{t_{i-1}}{f_{i-1}} f_{i-1} e^{-k}=$ $t_{i-1} e^{-k}$. Therefore, $t_{i}=t_{i-1}-t_{i-1} e^{-k}=t_{i-1}\left(1-e^{-k}\right)$. It is straightforward to see that the closed form expression for $t_{i}$ is

$$
t_{i}=t\left(1-e^{-k}\right)^{i-1}
$$

The value of $i$ for which $t_{i}=1$ is the expected value of the total number of frames $n$ that FRIP executes because $t_{i}=1$ means that there is only one unidentified tag in the population at the start of the $i^{\text {th }}$ frame and it will be identified without any collisions in the $i^{\text {th }}$ frame. Therefore, the value of $n$ can be obtained by equating $t_{n}$ with 1 , i.e., $t_{n}=t(1-$ $\left.e^{-k}\right)^{n-1}=1$, solving this expression for $n$, and subtracting 1 from it results in Equation (7). We subtract 1 because last frame is essentially a single slot with one unidentified tag. We don't count that frame towards total number of frames, instead we will add time equivalent to one singleton slot in the expected identification time to cater for the last single slot frame.

\section{E. Calculating Optimal Frame Size}

We substitute the expressions of expected values of $s_{i}, c_{i}$, $e_{i}$, and $n$ from Equations (4), (5), (6), and (7), respectively, into the expressions for Jain's fairness index and total identification time in Equations (2) and (3), respectively, to formulate the constraint optimization problem. We first derive the constraint that $k$ must not violate to ensure that observed value of $\mathcal{J}$ is greater than or equal to $\alpha$. This constraint is in the form of an upper bound on $k$ that $k$ should not exceed. After this, we derive a closed form expression for expected identification time. Using this closed form expression and the upper bound on $k$, we formulate the fair identification problem as a constraint optimization problem and solve it to calculate the optimal value of $k$ that minimizes the expected identification time under the constraint that $k$ does not exceed the upper bound. FRIP uses this optimal value of $k$ and divides the estimate of the size of unidentified tag population at the start of each frame by it to calculate the optimal size for each frame. Recall from Section I-C that FRIP obtains the estimate of size of unidentified tag population at the start of each frame by simply subtracting the number of tags successfully identified in the last frame from the number of unidentified tags at the start of the last frame. Theorem 1 calculates the value of the upper bound on $k$.

Theorem 1: The fairness achieved by FRIP in the number of transmissions per tag will be no less than $\alpha$ if the ratio, $k$, of number of unidentified tags to frame size is no greater than the upper bound $m$, where $m$ is given by the numerical solution of the following equation.

$\frac{\left(e^{m}(t-1) \ln \{A\}+\ln \{t\}\right)^{2}}{t\left(e^{m}\left(2 e^{m}-1\right)(t-1) \ln ^{2}\{A\}+2 e^{m} \ln \{A\} \ln \{t\}-\ln ^{2}\{t\}\right)}=\alpha$

where $A=1-e^{-m}$.

Proof: The observed value of the fairness index, $\mathcal{J}$, should satisfy the condition $\mathcal{J} \geq \alpha$. We first express the Jain's fairness index as a function of $k$ and discuss some of its properties. Then we show that if $k$ is less than or equal to $m$, the required fairness, $\alpha$, will be achieved i.e., the requirement $\mathcal{J} \geq \alpha$ will be satisfied.

By substituting the value of $s_{i}$ from Equation (4) into Equation (2), we get

$$
\mathcal{J}=\frac{\left(\sum_{i=1}^{n} i k f_{i} e^{-k}\right)^{2}}{t\left(\sum_{i=1}^{n} i^{2} k f_{i} e^{-k}\right)}
$$

According to Equation (8), $t_{i}=t\left(1-e^{-k}\right)^{i-1}$. For simplifying the presentation, we replace $1-e^{-k}$ by $r$. Thus, $t_{i}=t r^{i-1}$. As $k=\frac{t_{i}}{f_{i}} \Rightarrow t_{i}=k f_{i}$, therefore, $k f_{i}=t r^{i-1}$. By substituting this value of $k f_{i}$ into Equation (10) and simplifying, we get

$$
\mathcal{J}=\frac{e^{-k}\left(\sum_{i=1}^{n} i r^{i-1}\right)^{2}}{\sum_{i=1}^{n} i^{2} r^{i-1}}
$$




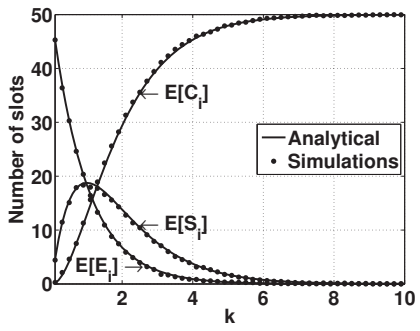

Figure $1 . \quad s_{i}, c_{i}, \& e_{i}$ vs. $k$

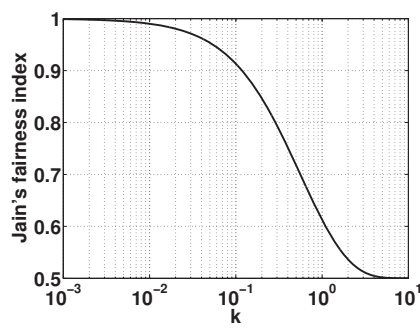

Figure 2. $\mathcal{J}$ vs. $k$

The values of the two summation terms of the equation above are given in the following two equations.

$$
\begin{gathered}
\sum_{i=1}^{n} i r^{i-1}=\frac{1}{(r-1)^{2}}\left\{1+n r^{n+1}-r^{n}(n+1)\right\} \\
\sum_{i=1}^{n} i^{2} r^{i-1}=\frac{1}{(r-1)^{3}}\left\{\begin{array}{l}
-1-r+n^{2} r^{n+2}+r^{n}(n+1)^{2} \\
\left.-r^{n+1}\left(2 n^{2}+2 n-1\right)\right\}
\end{array}\right.
\end{gathered}
$$

See appendix for the derivation of these two equations.

These two equations can be further simplified by putting $r^{n}=1 / t$. Next we show that $r^{n}$ is indeed equal to $1 / t$. As $r=1-e^{-k}$, raising both sides to the power $n$, using the value of $n$ from Equation (7), and taking natural log, we get

$$
\begin{aligned}
\ln \left\{r^{n}\right\} & =\ln \left\{\left(1-e^{-k}\right)^{n}\right\}=n \times \ln \left\{\left(1-e^{-k}\right)\right\} \\
& =-\frac{\ln \{t\}}{\ln \left\{1-e^{-k}\right\}} \times \ln \left\{\left(1-e^{-k}\right)\right\}=\ln \left\{\frac{1}{t}\right\} \\
\Rightarrow r^{n} & =\frac{1}{t}
\end{aligned}
$$

Substituting $r^{n}=1 / t$ and the value of $n$ from Equation (7) into Equations (12) and (13) and then substituting the resulting expressions of $\sum_{i=1}^{n} i r^{i-1}$ and $\sum_{i=1}^{n} i^{2} r^{i-1}$ into Equation (11), we get

$\mathcal{J}=\frac{\left(e^{k}(t-1) \ln \{r\}+\ln \{t\}\right)^{2}}{t\left(e^{k}\left(2 e^{k}-1\right)(t-1) \ln ^{2}\{r\}+2 e^{k} \ln \{r\} \ln \{t\}-\ln ^{2}\{t\}\right)}$

Figure 2 shows the plot of Jain's fairness index in the equation above as a function of $k$. We see that Jain's fairness index is a monotonically decreasing function of $k$. Therefore, replacing $k$ with $m$ in the equation above, equating it with $\alpha$, and solving it numerically, we get a unique value of the upper bound $m$. Due to the monotonically decreasing nature of Jain's fairness index, as long as the value of $k$ is less than or equal to $m$, the fairness achieved by FRIP will be greater than or equal to $\alpha$.

Next, in Theorem 2, we obtain a closed form expression for the expected identification time.

Theorem 2: Let $k$ be the ratio of number of unidentified tags in any given frame to the size of that frame. The expected time that FRIP takes to identify all tags in the population of size $t$ is given by the following equation.

$$
E[\mathcal{T}]=T_{s}+\frac{\left\{k T_{s}+\left(e^{k}-k-1\right) T_{c}+T_{e}\right\}\{t-1\}}{k}
$$

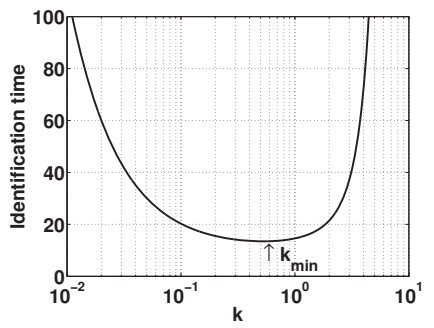

Figure 3. $E[\mathcal{T}]$ vs. $k$

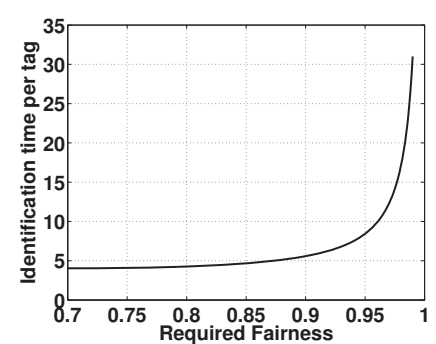

Figure 4. $E[\mathcal{T}] / t$ vs. $\alpha$
Proof: Applying expectation operator to both sides of Equation (3), we get

$$
\begin{aligned}
E[\mathcal{T}] & =T_{s}+E\left[\sum_{i=1}^{n}\left(s_{i} \times T_{s}+c_{i} \times T_{c}+e_{i} \times T_{e}\right)\right] \\
& =T_{s}+\sum_{i=1}^{n}\left(E\left[S_{i}\right] \times T_{s}+E\left[C_{i}\right] \times T_{c}+E\left[E_{i}\right] \times T_{e}\right)
\end{aligned}
$$

The extra $T_{s}$ in the equation above caters for the last single slot frame, as discussed in Section III-D. Substituting the values of $E\left[S_{i}\right], E\left[C_{i}\right]$, and $E\left[E_{i}\right]$ from Equations (4), (5), and (6) into the equation above, we get

$$
\begin{array}{r}
E[\mathcal{T}]=T_{s}+\sum_{i=1}^{n}( \\
\left(k f_{i} e^{-k}\right) \times T_{s}+\left(f_{i} e^{-k}\right) \times T_{e} \\
\left.+\left(f_{i}-k f_{i} e^{-k}-f_{i} e^{-k}\right) \times T_{c}\right)
\end{array}
$$

Recall that $r=1-e^{-k}$. Substituting $f_{i}$ by $t_{i} / k$ and in turn $t_{i}$ by $t r^{i-1}$ as per Equation (8), we get

$E[\mathcal{T}]=T_{s}+t\left\{T_{s} e^{-k}+T_{c} \frac{1-k e^{-k}-e^{-k}}{k}+T_{e} \frac{e^{-k}}{k}\right\} \sum_{i=1}^{n} r^{i-1}$

In the equation above, $\sum_{i=1}^{n} r^{i-1}$ is sum of a standard geometric series, which is equal to $\left(1-r^{n}\right) /(1-r)$. Using $r^{n}=1 / t$ from Equation (14), we get

$$
\sum_{i=1}^{n} r^{i-1}=\frac{1-1 / t}{1-\left(1-e^{-k}\right)}=e^{k}\left\{1-\frac{1}{t}\right\}
$$

Substituting the value of $\sum_{i=1}^{n} r^{i-1}$ from the equation above into Equation (16) results in Equation (15).

Now we finally define the constraint optimization problem as: calculate the optimal value of $k$ that minimizes the expected identification time, $E[\mathcal{T}]$, given by Equation (15) under the constraint $k \leq m$, where $m$ is obtained from the numerical solution of Equation (9).

We use Karush-Kuhn-Tucker (KKT) conditions to solve this optimization problem. To apply KKT conditions, we need an objective function that needs to be optimized and an inequality constraint. The objective function in this case is $E[\mathcal{T}]$, given by Equation (15), and the inequality constraint is $k-m \leq 0$. Equations (17) to (20) give the four KKT conditions that must be satisfied to obtain the optimal solution. 


$$
\begin{array}{rlrl}
\nabla E[\mathcal{T}]+\mu \nabla(k-m)=0, & & \text { Stationarity } \\
\mu(k-m)=0, & & \text { Slackness } \\
k-m \leq 0, & \text { Primal feasibility } \\
\mu \geq 0, & \text { Dual feasibility }
\end{array}
$$

where $\mu$ is called KKT multiplier.

From stationarity condition in Equation (17), we get

$$
\begin{array}{r}
\frac{d}{d k}\left[T_{s}+\frac{\left\{k T_{s}+\left(e^{k}-k-1\right) T_{c}+T_{e}\right\}\{t-1\}}{k}\right] \\
+\mu \frac{d}{d k}(k-m)=0 \\
\Rightarrow \mu=-\left[\frac{\left\{k e^{k} T_{c}-e^{k} T_{c}-T_{e}+T c\right\}\{t-1\}}{k^{2}}\right]
\end{array}
$$

From the slackness condition in Equation (18), we see that either $\mu=0$, or $k=m$. The optimal value of $k$ is $m$ if the value of $\mu$ evaluated from Equation (21) by using $k=m$ is not less than 0, i.e., the dual feasibility condition of Equation (20) is satisfied. If, however, the dual feasibility condition is not satisfied, i.e., the value of $\mu$ evaluated from Equation (21) by using $k=m$ is less than 0 , then $m$ is not the optimal value of $k$. In this case, we obtain the optimal value of $k$ by putting $\mu=0$ in Equation (21) and numerically solving it for $k$.

To get an intuitive insight into the process of finding the solution of this constraint optimization problem as described above, consider Figure 3 that plots Equation (15) as a function of $k$. Note from the figure that the identification time is a convex function of $k$, and thus has a minima at $k=k_{\min }$. When the value of $m$ calculated using Equation (9) is less than or equal to $k_{\min }$, then $m$ is the optimal value of $k$ because although the expected identification time could be reduced further by using a value of $k$ in the range $m \leq k \leq k_{\min }$, the value of fairness achieved in that case will not satisfy the requirement of $\mathcal{J} \geq \alpha$ because $\mathcal{J}$ is monotonically decreasing function of $k$ as seen in Figure 2. Relating this to KKT conditions, when $m \leq k_{\min }$, then using $k=m$ in Equation (21) results in $\mu \geq 0$, i.e., the dual feasibility condition is satisfied. Thus, in this case $m$ is indeed the optimal value of $k$. If, however, the value of $m$ calculated using Equation (9) is greater than $k_{\min }$, then although using $k=m$ will still satisfy the requirement $\mathcal{J} \geq \alpha$, the expected identification time will not be minimum. Therefore, instead of using $k=m$, we should use $k=k_{\min }$, because using $k=k_{\min }$ will result in smallest expected identification time and at the same time the requirement $\mathcal{J} \geq \alpha$ will still be satisfied because $\mathcal{J}$ is monotonically decreasing function of $k$. Relating this to KKT conditions, when $m>k_{\min }$, then using $k=m$ in Equation (21) results in $\mu<0$. Thus, in this case $m$ is not the optimal value of $k$. The optimal value is obtained by using $\mu=0$ in Equation (21) and solving it for $k$. The optimal value of $k$ obtained this way is equal to $k_{\min }$. Figure 4 shows that the expected identification time per tag, calculated by
Equation (15) with optimal $k$, increases with the increase in the required fairness, i.e., FRIP optimally trades-off the identification time to achieve the required fairness.

\section{F. Large Frame Size Implementation}

For large tag populations, the optimal frame size can exceed $2^{15}$, which is not supported by the C1G2 compliant RFID tags and readers. Next we present a strategy to execute frames with such sizes by using the SELECT command, standardized in the C1G2 standard. Using the SELECT command, a reader can broadcast a memory range and a bit mask to specify which tags should participate in the identification process. Each tag compares the bit mask with the bits in the specified range in its memory and participates in identification process only if the bit mask matches the specified bits in its memory. Using SELECT command, FRIP first divides the population into smaller groups of roughly equal sizes and then identifies the tags in each group independently using frames of allowed sizes.

To divide the whole population into groups of equal sizes, we leverage the fact that in large populations, the expected number of tags whose IDs have the least significant bit (LSB) of 0 is approximately the same as the expected number of tags whose IDs have the LSB of 1. Similarly, the expected number of tags whose IDs have the two LSBs of 00 is approximately the same as the expected number of tags whose IDs have the two LSBs of 01,10 , or 11, and so on. Therefore, a reader can divide the tag population into $2^{z}$ groups of roughly equal sizes by specifying appropriate masks for the memory range corresponding to the $z$ LSBs of tag IDs.

To execute $i^{\text {th }}$ frame, whose size, $f_{i}$, is greater than $f_{\max }=2^{15}$, the reader first determines the number of groups, $z$, that it should divide the tag population into by calculating $\left[\log _{2}\left\{f_{i} / f_{\max }\right\}\right]$. It then executes $2^{z}$ short frames of size $\left\lceil f_{i} / 2^{z}\right\rceil$ each instead of executing one long frame of size $f_{i}$. A short frame is a frame in which SELECT command is used and a long frame is a frame in which it is not. In each of the $2^{z}$ short frames, the reader uses SELECT command to specify a unique bit mask containing $z$ bits for the $z$ LSBs of tag IDs. In each short frame, on average, $t_{i} / 2^{z}$ tags participate, where $t_{i}$ is the number of unidentified tags at $i^{\text {th }}$ frame's start. Using the SELECT command in this way ensures that any given tag participates in exactly one short frame. Therefore, FRIP counts these $2^{z}$ short frames as one long frame. At the end of these $2^{z}$ short frames, FRIP performs re-estimation as described in Section I-C, and continues with the $i+1^{\text {st }}$ long frame.

Theorem 3 shows that executing $2^{z}$ short frames achieves same fairness as one long frame.

Theorem 3: Let the optimal frame size for the $i^{\text {th }}$ long frame be $f_{i}$, where $f_{i}>f_{\max }$. Let the number of unidentified tags at the start of this frame be $t_{i}$. The fairness achieved by executing $2^{z}$ short frames of size $\frac{f_{i}}{2^{z}}$ is the same as the fairness achieved by executing one long frame of size $f_{i}$. 
Proof: The value of fairness depends on the number of long frames, $n$, and number of singleton slots, $s_{i}$, in any long frame $i$, as seen in Equation (2). The value of $n$ does not change because $2^{z}$ short frames represent one long frame. Next we show that the expected value of $s_{i}$ obtained using $2^{z}$ short frames is the same as the expected value of $s_{i}$ obtained using one long frame, which is $s_{i}=k f_{i} e^{-k}$. When $2^{z}$ short frames are executed instead of one long frame, let we represent the number of singleton slots in the $j^{\text {th }}$ short frame with $s_{i j}$, where $1 \leq j \leq 2^{z}$. With the use of SELECT command as described earlier, the average number of tags that participate in the $j^{\text {th }}$ short frame is $t_{i} / 2^{z}$. As the size of each short frame is, $f_{i} / 2^{z}$, the value of ratio of number of unidentified tags that participate in each short frame to the size of frame is still $k$, i.e., $\frac{t_{i} / 2^{z}}{f_{i} / 2^{z}}=\frac{t_{i}}{f_{i}}=k$. Therefore, according to Equation (4), $s_{i j}=k \frac{f_{i}}{2^{z}} e^{-k}$. As $s_{i}=\sum_{j=1}^{2^{z}} s_{i j}$, therefore, $s_{i}=\sum_{j=1}^{2^{z}} k \frac{f_{i}}{2^{z}} e^{-k}=2^{z} \times k \frac{f_{i}}{2^{z}} e^{-k}=k f_{i} e^{-k}$. Thus, fairness remains the same.

\section{EXPERIMENTAL RESULTS}

We implemented and compared the performance of FRIP with nine prior tag identification protocols, namely the 3 nondeterministic (Aloha [21], BS [22], and ABS [23]), the 4 deterministic (TH [17], TW [24], ATW [25], and STT [26]), and the 2 hybrid protocols (MAS [27] and ASAP [28]). We did not compare FRIP with FNFRA [29] because the two protocols address different problems: FNFRA focuses on making reader scheduling process fair while FRIP focuses on making the tag identification process fair. Although FRIP is compatible with existing reader scheduling protocols, if we implement FRIP in conjuction with an existing reader scheduling protocol and compare its performance with FNFRA, we will essentially be comparing the fairness of the existing reader scheduling protocol with FNFRA, not FRIP.

Next, we first evaluate the fairness and identification time of FRIP and study how they are affected if the tag population is split because optimal frame size is greater than $2^{15}$. Then we present results from our side-by-side comparison of FRIP with existing protocols in terms of four metrics: (1) fairness, (2) number of tag transmissions, (3) energy consumption, and (4) identification time. In our simulations, we choose tag ID length to be the $\mathrm{C} 1 \mathrm{G} 2$ standard 64 bits. We performed our simulations on two different types of populations: tag populations with IDs distributed uniformly in the ID space and tag populations with IDs distributed non-uniformly in the ID space. For the uniform case, we range tag population sizes from 100 tags to 100, 000 tags. For the non-uniform case, we distribute tag population, containing 5000 tags, in blocks, where each block is a continuous sequence of tag IDs. We range block sizes from 5 to 1000. Our motivation for simulations on populations with tag IDs in blocks is that in some applications, such as supply chains, tag IDs often come in such blocks when they are manufactured. For each tag population size and block size, we run each protocol 10,000 times and then calculate the values of the four metrics. For example, we see how many times each tag transmits in 10,000 rounds of identification for a given protocol and then calculate the value of fairness. We do this to simulate the execution of identification protocols over a period of several days instead of a single identification round. In all the results reported for FRIP, we have incorporated the overhead of the estimation protocol that FRIP uses to obtain the initial estimate of tag population size.

\section{A. Evaluation of FRIP}

We evaluated FRIP for four different values of required fairness: $\alpha=0.80,0.90,0.95$, and 0.99 .

1) Fairness: Our results show that FRIP always achieves the required fairness irrespective of the size and type of the tag population. Figures 5(a) and 5(b) show the fairness achieved by FRIP in the number of transmissions per tag in uniform and non-uniform tag populations. The dashed horizontal lines in these two figures show the required fairness and the solid lines with markers show the actual fairness achieved by FRIP. We see that the solid lines always lie on or above the dashed lines, which shows that FRIP always achieves the required fairness.

2) Identification Time: Our results show that the identification time of FRIP obtained from simulations matches the theoretical minimum time obtained from Equation (15) using the optimal value of $k$, for all tag population sizes and distributions. Figure 6(a) shows the identification time of FRIP per tag for uniformly distributed tag populations using four different values of required fairness. Similarly, Figure 6(b) shows the identification time of FRIP per tag for nonuniformly distributed tag populations using four different values of required fairness. The durations of singleton, collision, and empty slots vary among the readers and are typically around $3 \mathrm{~ms}, 1.5 \mathrm{~ms}$, and $0.3 \mathrm{~ms}$, respectively, as reported in [17]. We have used these values of $3 \mathrm{~ms}$, $1.5 \mathrm{~ms}$, and $0.3 \mathrm{~ms}$ as durations of singleton, collision, and empty slots in our simulations. The dashed lines in these two figures represent the theoretical minimum identification times calculated from Equation (15) using the optimal values of $k$ for corresponding values of $\alpha$. The markers represent the average normalized identification times of FRIP obtained from simulations. We see that the markers always lie on their respective dashed lines, which shows that the identification time of FRIP obtained from simulations matches exactly with the minimum value of the identification time of FRIP calculated theoretically. This shows the correctness of the analytical model and that the independence assumption described in Section III-C does not create any problems. FRIP requires just 7.5 milliseconds of identification time per tag to achieve a fairness of 0.95 . Although the identification time increases with the increase in required fairness, it is still very small. For example, FRIP takes only one and a quarter minute to identify 10,000 tags when $\alpha=0.95$. 


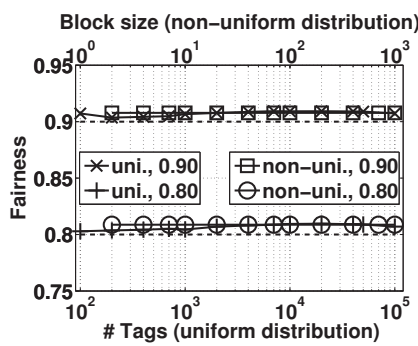

(a) $\alpha=0.80,0.90$

Figure 5

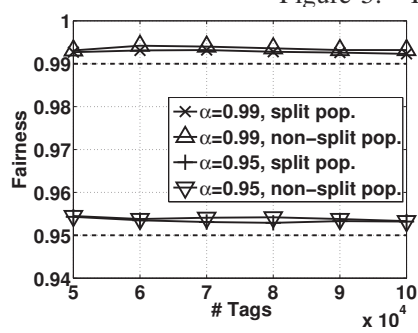

(a) Fairness

Figure 7 .

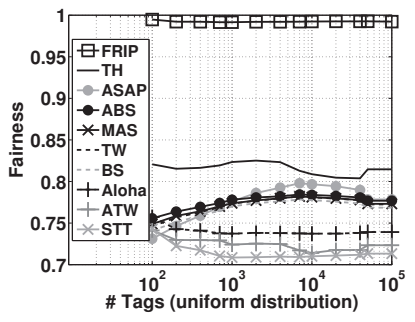

(a) Uniform distribution

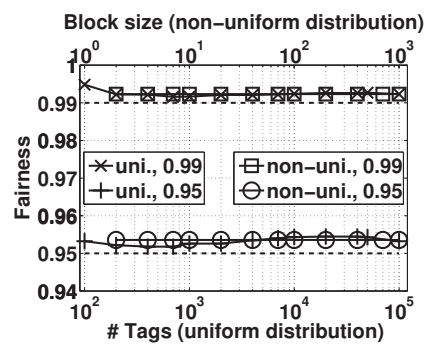

(b) $\alpha=0.95,0.99$

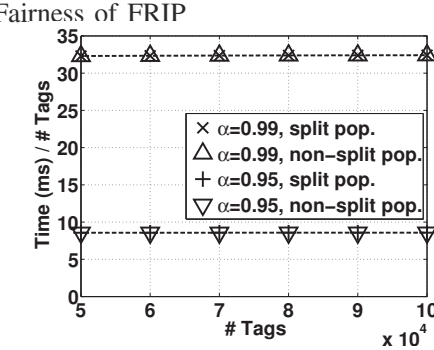

(b) Identification time

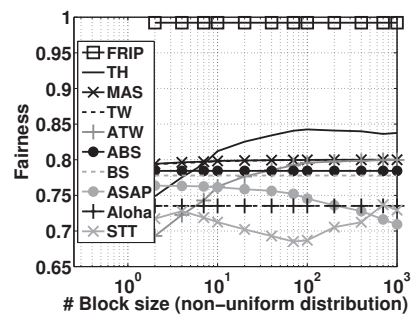

(b) Non-uniform distribution

3) Effect of Splitting Tag Population: Splitting the tag population due to optimal frame size greater than $2^{15}$ does not affect the fairness and identification time of FRIP. Figure 7(a) shows the fairness achieved by FRIP in the number of transmissions per tag when tag populations are split into smaller groups and when they are not split. The dashed horizontal lines in this figure show the required fairness and the solid lines with markers show the actual fairness achieved. We see that the solid lines always lie above the dashed lines for both cases i.e., when tag populations are not split and frame sizes greater than $2^{15}$ are used and when tag populations are split and frame sizes smaller than $2^{15}$ are used, as described in Section III-F. Figure 7(b) shows the normalized identification time of FRIP for split as well as non-split tag populations. The dashed lines in this figure represent the theoretical minimum identification times calculated from Equation (15) using the optimal values of $k$ for corresponding values of $\alpha$. The markers represent the average normalized identification times of FRIP with split and non-split populations. We see that the markers for split and non-split populations overlap and always lie on their respective dashed lines.

4) Effect of Error in Population Size Estimate: FRIP tolerates an error of upto $15 \%$ in population size estimate

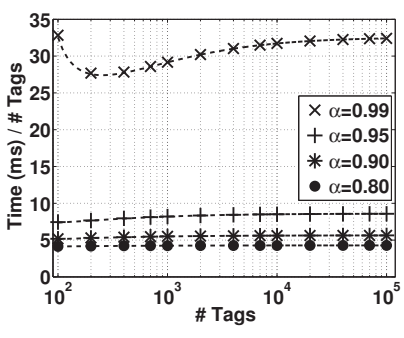

(a) Uniform distribution

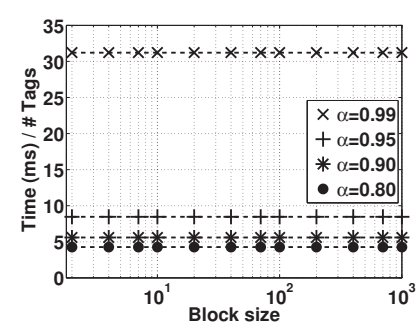

(b) Non-uniform distribution Figure 6. Identification Time of FRIP

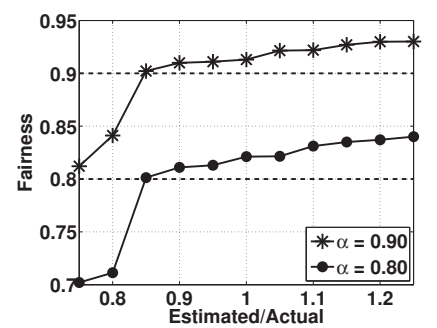

(a) $\alpha=0.80,0.90$

Figure 8. Effect of error in population size estimate

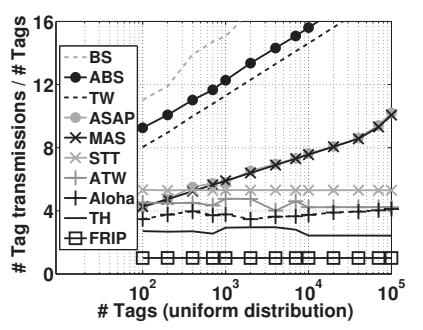

(a) Uniform distribution

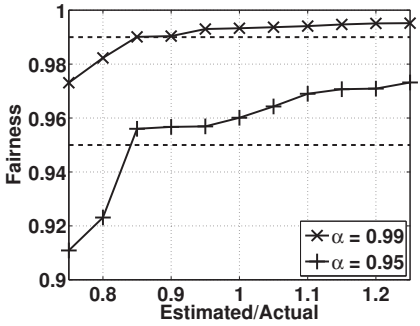

(b) $\alpha=0.95,0.99$

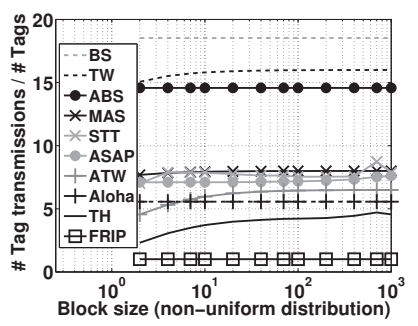

(b) Non-uniform distribution
Figure 10. Normalized Transmissions Comparison

before its fairness drops below the required fairness. Figures 8(a) and 8(b) plot the fairness achieved by FRIP for different values of errors in population size estimates. The horizontal axes in these figures represent the ratio of estimated tag population size to actual tag population size. The dashed horizontal lines show the required fairness and the solid lines with markers show the actual fairness achieved. We see that the solid lines always cross the dashed lines at or before the ratio of 0.85 . We also observe that as the estimated tag population size gets larger than the actual tag population size, FRIP achieves better fairness than required because it uses larger frame sizes. This means that the improved fairness comes at the cost of slow identification. Therefore, it is important that the estimate of tag population size is close to its actual size. In our simulations, we configure ART such that the estimates lie within $\pm 5 \%$ of the actual value.

\section{B. Comparison with Existing Protocols}

We compare FRIP with existing protocols in terms of following four metrics: fairness, normalized number of tag transmissions, normalized power consumption, and normalized identification time. The term normalized means that we report these metrics on per tag basis, i.e., we first calculate the value of the metric for the whole population and then divide it by the size of the tag population. Compared to 
non-normalized values, normalized values of metrics give a better idea of the effect of population size on the metric. For example, the non-normalized value of the identification time for the whole population is bound to increase with the increase in tag population size, and thus, does not indicate whether the identification time per tag stays constant or changes with the change in population size.

1) Fairness: Our results show that FRIP achieves higher fairness than all existing protocols. This is because FRIP takes required fairness as input and can achieve arbitrarily high fairness (such as 0.99) by optimally trading-off identification time for it. In contrast, the existing protocols do not take the required amount of fairness as input. Figures 9(a) and 9(b) show the fairness achieved by FRIP and the nine existing protocols for populations with uniform and nonuniform distributions, respectively. Among the existing protocols, TH achieves the highest fairness, which is bounded above by 0.84 .

2) Normalized Tag Transmissions: Our results show that the average number of transmissions per tag are the fewest for FRIP compared to existing protocols. For example, when FRIP is configured for a required fairness of 0.99 , it reduces the normalized tag transmissions compared to the best prior C1G2 compliant nondeterministic, deterministic, and hybrid tag identification protocols by an average of $3.74,2.62$, and 6.85 times, respectively, for uniformly distributed tag populations, and by an average of $5.50,3.88$, and 7.86 times, respectively, for non-uniformly distributed tag populations. Fewer transmissions per tag means lesser power consumption at the tags, which increases the battery life. Figures 10(a) and 10(b) show the normalized tag transmissions of all protocols for uniformly and non-uniformly distributed tag populations, respectively.

3) Normalized Energy Consumption: Our results show that the tags consume least amount of energy when the reader executes FRIP. Although we have already shown that the number of transmissions per tag are the fewest for FRIP, this does not guarantee that the overall power consumed per tag is also the smallest, because empty slots also consume some power. A tag consumes approximately $1 \mathrm{~mW}$ of power during singleton slots and $35 \mathrm{~mW}$ of power during empty and collision slots [27]. Using these values of power, when FRIP is configured for a required fairness of 0.99 , it reduces the power consumption per tag compared to the best prior $\mathrm{C} 1 \mathrm{G} 2$ compliant nondeterministic, deterministic, and hybrid tag identification protocols by an average of $15.2 \%$, $11 \%$, and $28.7 \%$, respectively, for uniformly distributed tag populations, and by an average of $14.4 \%, 11.3 \%$, and $384 \%$, respectively, for non-uniformly distributed tag populations. Figures 11(a) and 11(b) show the normalized power consumption of all protocols for uniformly and non-uniformly distributed tag populations, respectively. FRIP consumes the least amount of power while achieving the highest fairness.

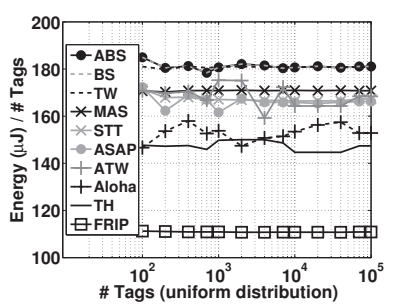

(a) Uniform distribution

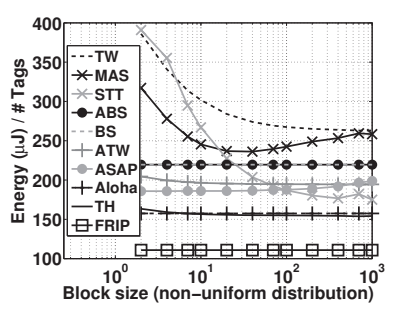

(b) Non-uniform distribution
Figure 11. Normalized Energy Comparison

4) Normalized Identification Time: Our results show that identification time of FRIP is smaller than all existing protocols, except one, when the reader executes FRIP with the required fairness equal to what the corresponding existing protocols achieve. As the identification time of FRIP is a function of required fairness, for a fair comparison, we first determined the fairness value that each protocol achieves, and then executed FRIP using those values as the required fairness. Table II reports the fairness and identification times per tag for all existing protocols in the second and third columns, respectively. It also reports the identification times per tag of FRIP in the fourth column when executed for the required fairness values in the second column. FRIP is $21.13 \%$ and $13.19 \%$ faster than the best prior $\mathrm{C} 1 \mathrm{G} 2$ compliant nondeterministic and hybrid tag identification protocols, respectively. FRIP is faster than all deterministic protocols as well except $\mathrm{TH}$, which is $13.9 \%$ faster than FRIP. Although TH is faster, it cannot achieve an arbitrarily high fairness.

Table II

PER TAG IDENTIFICATION TIME COMPARISON

\begin{tabular}{|c|c|c|c|c|}
\hline Protocol & Fairness & $\begin{array}{c}\text { Protocol } \\
(\mathrm{ms} / \mathrm{Tag})\end{array}$ & $\begin{array}{c}\text { FRIP } \\
(\mathrm{ms} / \mathrm{Tag})\end{array}$ & $\begin{array}{c}\text { Improve- } \\
\text { ment }(\%)\end{array}$ \\
\hline STT & 0.71 & 4.64 & 4.05 & 14.57 \\
\hline ATW & 0.72 & 4.37 & 4.05 & 07.90 \\
\hline Aloha & 0.74 & 4.93 & 4.07 & 21.13 \\
\hline ABS & 0.78 & 5.30 & 4.17 & 27.10 \\
\hline BS & 0.78 & 5.30 & 4.17 & 27.10 \\
\hline MAS & 0.78 & 4.72 & 4.17 & 13.19 \\
\hline TW & 0.78 & 5.30 & 4.17 & 27.10 \\
\hline ASAP & 0.79 & 4.59 & 4.22 & 08.77 \\
\hline TH & 0.82 & 3.78 & 4.39 & -13.90 \\
\hline
\end{tabular}

\section{CONCLUSION}

The key technical contribution of this paper is in proposing a fair identification protocol for reading the IDs of all tags in an RFID tag population such that the number of transmissions per tag are equal. The key technical depth of this paper is in the mathematical development of the theory that FRIP is based upon. The solid theoretical underpinning of FRIP ensures that the actual fairness that it achieves is greater than or equal to the required fairness. We have developed a novel technique that FRIP uses to execute large frame sizes to ensure compliance with the $\mathrm{C} 1 \mathrm{G} 2$ standard. We have presented a comprehensive evaluation of FRIP and its side-by-side comparisons with nine major prior 
tag identification protocols. Our experimental results show that FRIP significantly outperforms all prior identification protocols, even those that are not $\mathrm{C} 1 \mathrm{G} 2$ compliant, for not only the fairness but also the metrics such as number of transmissions per tag and power consumption.

\section{REFERENCES}

[1] J. Collins and S. E. Zweig, "Sensing a product's shelf life," RFID Journal.

[2] A. Nemmaluri, M. D. Corner, and P. Shenoy, "Sherlock: Automatically locating objects for humans," in Proc. MobiSys, 2008, pp. 187-198.

[3] B. Nath, F. Reynolds, and R. Want, "RFID technology and applications," Proc. IEEE Pervasive Computing, vol. 5, pp. 22-24, 2006.

[4] M. Roberti, "Wal-mart relaunches EPC RFID effort, starting with men's jeans and basics," RFID Journal, 2010.

[5] C. Swedberg, "Honeywell aerospace tags parts for airbus," RFID Journal.

[6] — , "Dutch researchers focus on RFID-based sensors for monitoring apnea, epilepsy," RFID Journal, 2007.

[7] M. C. O'Connor, "HP kicks off US RFID demo center," RFID Journal.

[8] "Sprinkling RFID sensor tags from the sky," rfidinjapan. wordpress.com/2006/03/20/sprinkling-rfid-sensor-tags-from-the-sky

[9] “Tempcorder 2.4GHz Active RFID Tag Series," http://www. hk-rfid.com/home/news201211b_Tempcorder\%20Case_ 121109.pdf.

[10] S. Ohkubo and K. Takiishi, "Technologies to reduce power consumption of active RFID tags," NTT DoCoMo Journal, vol. 8, no. 1, 2006.

[11] A. Amanna, A. Agrawal, and M. Manteghi, "Active RFID for enhanced railway operations," in Proc. ASME Rail Transport Division Conf. , 2010.

[12] "TI's RFID tagging system tracks and acoustic transponders in offshore seismic exploration," Assembly Automation, vol. 20 , no. 1 .

[13] "Tracking \& tracing at BMW," http://www.brooks-rfid.com/ trackingtracing-at-bmw.html

[14] M. C. O'Connor, "Boeing wants dreamliner parts tagged," RFID Journal

[15] R. K. Jain, D.-M. W. Chiu, and W. R. Hawe, "A quantitative measure of fairness and discrimination for resource allocation in shared computer systems," Digital Equipment Corporation, Tech. Rep., 1984.

[16] Radio-Frequency Identity Protocols C1G2 UHF RFID Protocol for Communications at $860 \mathrm{MHz}-960 \mathrm{MHz}$, EPCGlobal Inc, 2013.

[17] M. Shahzad and A. X. Liu, "Probabilistic optimal tree hopping for RFID identification," in Proc. ACM SIGMETRICS, 2013.

[18] - "Every bit counts - fast and scalable RFID estimation," in Proc. ACM MobiCom, 2012, pp. 365-376.

[19] Z. Zhou, H. Gupta, S. R. Das, and X. Zhu, "Slotted scheduled tag access in multi-reader RFID systems," in Proc. IEEE ICNP, 2007, pp. 61-70.
[20] S. Tang, J. Yuan, X.-Y. Li, G. Chen, Y. Liu, and J. Zhao, "Raspberry: A stable reader activation scheduling protocol in multi-reader RFID systems," in Proc. IEEE ICNP, 2009, pp. 304-313.

[21] B. Zhen, M. Kobayashi, and M. Shimizu, "Framed ALOHA for multiple RFID objects identification," IEICE Trans. on Communications, 2005.

[22] J. I. Capetanakis, "Tree algorithms for packet broadcast channels," IEEE Transactions on Information Theory, vol. 25, pp. 505-515, 1979.

[23] J. Myung and W. Lee, "Adaptive splitting protocols for RFID tag collision arbitration," in Proc. ACM MobiHoc, 2006, pp. 202-213.

[24] C. Law, K. Lee, and K.-Y. Siu, "Efficient memoryless protocol for tag identification," in Proc. Discrete Algorithms and Methods, 2000.

[25] A. S. Tanenbaum, Computer Networks. Prentice-Hall, 2002.

[26] L. Pan and H. Wu, "Smart trend-traversal: A low delay and energy tag arbitration protocol for large RFID systems," in Proc. INFOCOM, 2009.

[27] V. Namboodiri and L. Gao, "Energy-aware tag anticollision protocols for RFID systems," in Proc. IEEE PerCom, 2007, pp. 23-36.

[28] C. Qian, Y. Liu, H. Ngan, and L. M. Ni, "ASAP: Scalable identification and counting for contact less RFID systems," in Proc. ICDCS, 2010.

[29] R. Ferrero, F. Gandino, B. Montrucchio, and M. Rebaudengo, "Fair anti-collision protocol in dense RFID networks," in Proc. of 3rd Int. EURASIP Workshop on RFID Technology, 2010, pp. 101-105.

[30] L. Chen, I. Demirkol, and W. Heinzelman, "Token MAC: a fair MAC protocol for passive RFID systems," in Proc. IEEE GLOBECOM, 2011

[31] R. Sámano-Robles and A. Gameiro, "Throughput, stability and fairness of RFID anti-collision algorithms with tag cooperation," Int. Journal On Advances in Telecom., vol. 5, pp. 229-238, 2012.

[32] H. Vogt, "Efficient object identification with passive RFID tags," Pervasive Computing, vol. 2414, pp. 98-113, 2002.

[33] M. Kodialam and T. Nandagopal, "Fast and reliable estimation schemes in RFID systems," in Proc. MobiCom, 2006, pp. 322-333.

[34] C. Bordenave, D. McDonald, and A. Proutiere, "Performance of random medium access control, an asymptotic approach," in Proc. ACM SIGMETRICS, 2008.

\section{APPENDIX}

The expanded form of left hand side of Equation (12) is $\sum_{i=1}^{n} i r^{i-1}=1 r^{0}+2 r^{1}+3 r^{2}+\ldots+n r^{n-1}$. Multiplying it with $r$, and subtracting the resultant from $\sum_{i=1}^{n} i r^{i-1}$, we get

$$
\sum_{i=1}^{n} i r^{i-1}-r \sum_{i=1}^{n} i r^{i-1}=\sum_{i=1}^{n} r^{i-1}-n r^{n}
$$

Substituting $\sum_{i=1}^{n} r^{i-1}$ by $\left(1-r^{n}\right) /(1-r)$ in the equation above and rearranging, we get Equation (12). Equation (13) can be derived using same steps. 